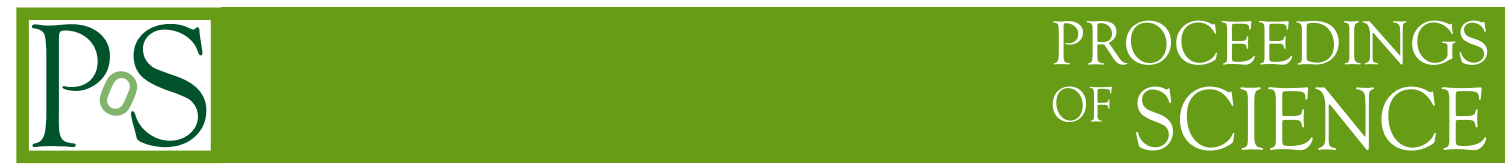

\title{
Monopoles, topology of the Standard Model, and unification of interactions at Tev scale
}

\author{
Mikhail Zubkov*i \\ E-mail: zubkov@itep.ru
}

It is shown that unification of strong and Electroweak interactions at Tev scale may lead to appearance of topologically stable monopoles with masses of the order of $40 \mathrm{Tev}$. Those monopoles may play an important role in the early Universe, at finite temperature. They may even be condensed at high enough temperature. The lattice model for investigation of this phenomenon is presented.

The XXV International Symposium on Lattice Field Theory

July 30 - August 42007

Regensburg, Germany

\footnotetext{
* Speaker.

${ }^{\dagger}$ ITEP, B.Cheremushkinskaya 25, Moscow, 117259, Russia
} 


\section{Introduction}

Due to the so-called Hierarchy problem [1] at the energy scale of about 1 Tev new physics is expected to appear. Naturalness of the theory [1] leads to the requirement that the ultraviolet cutoff $\Lambda$ is of the order of $1 \mathrm{TeV}$. It is worth mentioning that this problem appears also in lattice nonperturbative study (see, for example, [2]). Thus at the Tev scale some other theory should appear, which incorporates Standard Model as a low energy approximation.

There are several patterns of unification of interactions, which were considered up to now. Among them there are at least three examples, in which gauge group of the Standard Model is extended already at the Tev scale. Namely, in the so - called Little Higgs models [3, 4] $S U(2) \times$ $U(1)$ subgroup is embedded into a larger group, which is gauged partially. The correspondent symmetry is broken at a few Tev.

The second example is the Extended Technicolor Theory. The idea is to construct the new Tev scale theory basing on the analogy with QCD $[5,6,7,8]$. The new Nonabelian gauge interaction is added with the scale $\Lambda_{T C} \sim 1 \mathrm{Tev}$, where $\Lambda_{T C}$ is the analogue of $\Lambda_{Q C D}$. This new interaction is called technicolor. The correspondent new fermions are called technifermions. Breaking of the chiral symmetry in technicolor theory causes Electroweak symmetry breaking. In order to make Standard Model fermions massive extra gauge interaction is added, which is called Extended technicolor (ETC) $[5,8]$. In this new gauge theory the Standard Model fermions and technifermions enter the same representation of Extended technicolor group. There is a great number of ETC models. In particular, there exist models such that the ETC gauge group unifies strong and Electroweak interactions at Tev scale.

The third example is the so-called Petite Unification (see, for example, $[9,10]$ and references therein). In the correspondent models the gauge symmetry of the Standard Model is extended to a larger one at the Tev scale. The resulting models have two different coupling constants correspondent to strong and Electroweak interactions unlike Grand Unified models, in which there is only one coupling constant and the unification is achieved at the GUT scale $10^{15} \mathrm{Gev}$.

Long time ago it was recognized that the spontaneous breakdown of $S U(5)$ symmetry in the Unified model actually leads to the gauge group $S U(3) \times S U(2) \times U(1) / Z_{6}$ instead of the conventional $S U(3) \times S U(2) \times U(1)$ (see, for example, [11] and references therein). The appearance of the additional $Z_{6}$ symmetry in the fermion and Higgs sectors of the Standard Model itself was recovered later within the lattice field theory $[12,13,14,15]$. Independently $Z_{6}$ symmetry in the Higgs sector of the Standard Model was considered in [16]. The emergence of $Z_{6}$ symmetry in technicolor models was considered in [17].

Due to the $Z_{6}$ symmetry the gauge group of the Standard Model is either $S U(3) \times S U(2) \times U(1)$ or $S U(3) \times S U(2) \times U(1) / \mathscr{Z}$, where $\mathscr{Z}$ is equal to $Z_{6}$, or to one of its subgroups: $Z_{3}$ or $Z_{2}$. It would be important to know is there any difference between the correspondent models or not.

On the level of perturbation expansion those versions of the Standard Model are identical. Here we take into account that the Standard Model describes nature only up to the energies of about a few Tev. This can have an effect on the topology of the Standard Model. Namely, there may appear small regions of sizes of the order of $1 \mathrm{Tev}^{-1}$, where the conventional fields of the Standard Model are not defined. These regions may represent monopoles of the unified theory. 
Then different versions of the Standard Model lead to different monopole contents of the models that describe Tev - scale physics.

At zero temperature unified gauge group is broken to the Standard Model gauge group. However, at the temperatures higher than the critical temperature $T_{c} \sim 1 \mathrm{Tev}$ this symmetry should be restored. Basing on the analogy with Nambu monopoles [15] we expect that the monopoles of the models, which describe Tev - scale physics, may be condensed at $T>T_{c}$. In order to investigate this phenomenon we suggest to consider the lattice toy model based on one of the Petite Unification models.

\section{Monopoles of the unified theory}

Let us now fix the closed surface $\Sigma$ in 4-dimensional space $R^{4}$. For any closed loop $\mathscr{C}$, which winds around this surface, we may calculate the Wilson loops $\Gamma=\operatorname{Pexp}\left(i \int_{\mathscr{C}} C^{\mu} d x^{\mu}\right), U=$ $\operatorname{Pexp}\left(i \int_{\mathscr{C}} A^{\mu} d x^{\mu}\right)$, and $e^{i \theta}=\exp \left(i \int_{\mathscr{C}} B^{\mu} d x^{\mu}\right)$, where $C, A$, and $B$ are correspondingly $S U$ (3), $S U(2)$ and $U(1)$ gauge fields of the Standard Model. In the usual realization of the Standard Model with the gauge group $S U(3) \times S U(2) \times U(1)$ such Wilson loops should tend to unity, when the length of $\mathscr{C}$ tends to zero $(|\mathscr{C}| \rightarrow 0)$. However, in the $S U(3) \times S U(2) \times U(1) / \mathscr{Z}$ gauge theory the following values of the Wilson loops are allowed at $|\mathscr{C}| \rightarrow 0$ :

$$
\begin{aligned}
\Gamma & =\operatorname{Pexp}\left(i \int_{\mathscr{C}} C^{\mu} d x^{\mu}\right)=e^{N \frac{2 \pi i}{3}} \\
U & =\operatorname{Pexp}\left(i \int_{\mathscr{C}} A^{\mu} d x^{\mu}\right)=e^{-N \pi i} \\
e^{i \theta} & =\exp \left(i \int_{\mathscr{C}} B^{\mu} d x^{\mu}\right)=e^{N \pi i}
\end{aligned}
$$

where $N=0,1,2,3,4,5$ for $\mathscr{Z}=Z_{6}, N=0,2,4$ for $\mathscr{Z}=Z_{3}$, and $N=0,3$ for $\mathscr{Z}=Z_{2}$. Then the surface $\Sigma$ may carry $Z_{2}$ flux $\pi[N \bmod 2]$ for $\mathscr{Z}=Z_{2}, Z_{6}$. It also may carry $Z_{3}$ flux $\frac{2 \pi[N \bmod 3]}{3}$ for $\mathscr{Z}=Z_{3}, Z_{6}$.

Any configuration with the singularity of the type (2.1) could be eliminated via a singular gauge transformation. Therefore the appearance of such configurations in the theory with the gauge group $S U(3) \times S U(2) \times U(1) / \mathscr{Z}$ does not influence the dynamics.

Now let us consider an open surface $\Sigma$. Let the small vicinity of its boundary $U(\partial \Sigma)$ represent a point - like soliton state of the unified theory. This means that the fields of the Standard Model are defined now everywhere except $U(\partial \Sigma)$. Let us consider such a configuration, that for infinitely small contours $\mathscr{C}$ (winding around $\Sigma$ ) the mentioned above Wilson loops are expressed as in (2.1). For $N \neq 0$ it is not possible to expand the definition of such a configuration to $U(\partial \Sigma)$. However, this could become possible within the unified model if the gauge group of the Standard Model $S U(3) \times$ $S U(2) \times U(1) / \mathscr{Z}$ is embedded into the simply connected group $\mathscr{H}$. This follows immediately from the fact that any closed loop in such $\mathscr{H}$ can be deformed smoothly to a point and this point could be moved to unity. Actually, for such $\mathscr{H}$ we have $\pi_{2}(\mathscr{H} /[S U(3) \times S U(2) \times U(1) / \mathscr{Z}])=$ $\pi_{1}(S U(3) \times S U(2) \times U(1) / \mathscr{Z})$. This means that in such unified model the monopole-like soliton states are allowed. The configurations with (2.1) and $N \neq 0$ represent fundamental monopoles of 
the unified model ${ }^{1}$. The other monopoles could be constructed of the fundamental monopoles as of building blocks. In the unified model, which breaks down to the SM with the gauge group $S U(3) \times S U(2) \times U(1)$ such configurations for $N \neq 0$ are simply not allowed. This gives us the way to distinguish between the two versions of the Standard Model.

The unified model, which breaks down to the $\mathrm{SM}$ with the gauge group $S U(3) \times S U(2) \times U(1)$ also contains monopoles because $\pi_{2}(\mathscr{H} /[S U(3) \times S U(2) \times U(1)])=\pi_{1}(S U(3) \times S U(2) \times U(1))=$ $Z$. They correspond to the Dirac strings with $\int_{\mathscr{C}} B^{\mu} d x^{\mu}=6 \pi K, K \in Z$ and should be distinguished from the monopoles (for $N=1,2,3,4,5$ ) of the SM with the additional discrete symmetry via counting their magnetic fluxes.

Using an analogy with t'Hooft - Polyakov monopoles[19] we can estimate masses of the mentioned monopoles to be of the order of $N \sqrt{\frac{4 \pi}{\alpha}} \Lambda \sim 40 N \mathrm{Tev}$, where $\Lambda \sim 1 \mathrm{Tev}$ is the scale of the breakdown and $\alpha$ is the fine structure constant $\left(\alpha\left(M_{Z}\right) \sim \frac{1}{128}\right)$ (see, for example, [20], where monopoles were considered for an arbitrary compact simple gauge group in the BPS limit). Here $N=6$ for monopoles of the conventional Standard Model.

\section{Monopoles in Petite Unification models}

In order to illustrate the emergence of the additional $Z_{3}$ and $Z_{2}$ symmetries in the Standard Model we consider Petite Unification of strong and Electroweak interactions discussed in $[9,10]$. In the mentioned papers three possibilities to construct the unified theory at Tev were distinguished among a number of various ones. Namely, let us consider the unified group to be the product of $S U(4)_{P S}$ and $S U(N)^{k}$, where $S U(4)_{P S}$ unifies lepton number with color as in Pati-Salam models [21]. In the theory there are two independent couplings $\alpha_{S}$ and $\alpha_{W}$ correspondent to the two groups mentioned above. Then if we require that the spontaneous breakdown of $S U(4)_{P S} \otimes S U(N)^{k}$ happens at a Tev scale we are left with the three possibilities: $\operatorname{PUT}_{0}(N=2, k=4) ; \operatorname{PUT}_{1}(N=2, k=$ $3) ; \operatorname{PUT}_{2}(N=3, k=2)$. The other choices of $N$ and $k$ cannot provide acceptable values of coupling constants at the Electroweak scale. It should be mentioned here that $\mathrm{PUT}_{0}$ seems to be excluded due to the extremely high value of branching ratio for the process $K_{L} \rightarrow \mu e$.

It will be useful to represent the breakdown pattern correspondent to the models $P U T_{1}, P U T_{2}$ in terms of the loop variables $\Gamma, U$, and $\theta$ calculated along the arbitrary closed contour $\mathscr{C}$.

In $\mathrm{PUT}_{2}$ at the Electroweak scale $S U(4)_{P S} \otimes S U(3)^{2}$ parallel transporter $\Omega$ along the contour $\mathscr{C}$ is expressed through $\Gamma, U$, and $\theta$ as follows:

$$
\Omega=\left(\begin{array}{cc}
\Gamma^{+} e^{\frac{2 i \theta}{3}} & 0 \\
0 & e^{-2 i \theta}
\end{array}\right) \otimes\left(\begin{array}{ccc}
e^{\frac{-4 i \theta}{3}} & 0 & 0 \\
0 & e^{\frac{2 i \theta}{3}} & 0 \\
0 & 0 & e^{\frac{2 i \theta}{3}}
\end{array}\right) \otimes\left(\begin{array}{cc}
U e^{-\frac{i \theta}{3}} & 0 \\
0 & e^{\frac{2 i \theta}{3}}
\end{array}\right)
$$

From (3.1) it is straightforward that values (2.1) of the Wilson loops $\Gamma, U$, and $e^{i \theta}$ with $N=$ $0,3 \in Z_{2}$ lead to $\Omega=\mathbf{1}$. The field strength of the $S U(4)_{P S} \otimes S U(3)^{2}$ gauge field is expressed

\footnotetext{
${ }^{1}$ Actually these configurations were already considered (see, for example [11], where they represent fundamental monopoles of the $S U(5)$ unified model). However, in [11] it was implied that such soliton states could appear with the masses of the order of GUT scale $\left(10^{15} \mathrm{Gev}\right)$. In our case the appearance of such objects is expected already at the energies compared to $1 \mathrm{Tev}$ because we consider the unified model, in which $\mathscr{H}$ is broken to the gauge group of the Standard Model already at this scale.
} 
through $\Omega$ calculated along the infinitely small contour. Then the pure gauge field action in the low energy limit (at the Electroweak scale) is invariant under an additional $Z_{2}$ symmetry. This means that in $\mathrm{PUT}_{2}$ actual breakdown pattern is $S U(4)_{P S} \otimes S U(3)^{2} \rightarrow S U(3) \times S U(2) \times U(1) / Z_{2}$ and not $S U(4)_{P S} \otimes S U(3)^{2} \rightarrow S U(3) \times S U(2) \times U(1)$. Therefore, we expect $S U(2) / Z_{2}$ monopoles to exist in this unified model with the masses of the order of $120 \mathrm{Tev}$.

Here we used the values of Electroweak charges calculated in [9] in order to represent the breakdown pattern in a form useful for our purposes. One can check directly that the gauge group element of the form (3.1) acts appropriately on the Standard Model fermions arranged in the representations listed in [9]. The same check could be performed also for the model $\mathrm{PUT}_{1}$ considered below.

In $\mathrm{PUT}_{1}$ at the Electroweak scale $S U(4)_{P S} \otimes S U(2)^{3}$ parallel transporter $\Omega$ along the contour $\mathscr{C}$ is expressed as follows:

$$
\Omega=\left(\begin{array}{cc}
\Gamma^{+} e^{\frac{2 i \theta}{3}} & 0 \\
0 & e^{-2 i \theta}
\end{array}\right) \otimes U \otimes\left(\begin{array}{cc}
e^{-i \theta} & 0 \\
0 & e^{i \theta}
\end{array}\right) \otimes\left(\begin{array}{cc}
e^{i \theta} & 0 \\
0 & e^{-i \theta}
\end{array}\right)
$$

It is straightforward that values (2.1) of the Wilson loops $\Gamma, U$, and $e^{i \theta}$ with $N=0,2,4 \in$ $Z_{3}$ lead to $\Omega=1$. This means that in $\mathrm{PUT}_{1}$ actual breakdown pattern is $S U(4)_{P S} \otimes S U(2)^{3} \rightarrow$ $S U(3) \times S U(2) \times U(1) / Z_{3}$ and not $S U(4)_{P S} \otimes S U(2)^{3} \rightarrow S U(3) \times S U(2) \times U(1)$. Thus, $S U(3) / Z_{3}$ monopoles should exist in this unified model with the masses of the order of $80 \mathrm{Tev}$.

\section{Lattice model for qualitative investigation of the monopoles}

Basing on the analogy with Nambu monopoles [15] we expect that the monopoles of the models, which describe Tev - scale physics, may be condensed at $T>T_{c}$. In order to investigate this phenomenon we construct the lattice toy model based on $\mathrm{PUT}_{2}$.

We simplify $\mathrm{PUT}_{2}$ in such a way that the resulting model has the gauge group $S U(3)$. At low energies parallel transporter $\Omega \in S U(3)$ along the contour $\mathscr{C}$ is expressed through $U \in S U(2)$, and $\theta \in U(1)$ as follows:

$$
\Omega=\left(\begin{array}{cc}
U e^{-\frac{i \theta}{3}} & 0 \\
0 & e^{\frac{2 i \theta}{3}}
\end{array}\right)
$$

In this theory the breakdown pattern is $S U(3) \rightarrow S U(2) \times U(1) / Z_{2}$. Therefore, $Z_{2}$ monopoles exist. The lattice model contains link $S U(3)$ field $\Omega$ and the adjoint scalar $\Phi \in \operatorname{su}(3)$ defined on sites. The potential for the scalar field may be considered in its simplest form in the London limit. In this case the action has the form

$$
\begin{aligned}
S_{g}= & \beta \sum_{\text {plaquettes }}\left(\left(1-\frac{1}{3} \operatorname{Re} \operatorname{Tr} \Omega_{p}\right)+\right. \\
& +\gamma \sum_{x y}\left(1-\frac{1}{2} \operatorname{Re} \operatorname{Tr}\left(\Omega_{x y} \lambda_{8} \Omega^{+} \lambda_{8}\right)\right),
\end{aligned}
$$

where the plaquette variables are defined as $\Omega_{p}=\Omega_{x y} \Omega_{y z} \Omega_{w z}^{*} \Omega_{x w}^{*}$ for the plaquette composed of the vertices $x, y, z, w . \beta$ is usual $S U(3)$ coupling while $\gamma$ is proportional to squared vacuum expectation value of the scalar field. 
The hypercharge $U(1)$ gauge field in the theory can be expressed through $\Omega$ as follows: $\theta=$ $\frac{3}{2} \operatorname{Arg} \Omega_{33}$. In lattice theory monopole classical solution should be formed around the Dirac string, which is represented by the integer-valued field defined on the dual lattice

$$
\Sigma=\frac{1}{2 \pi}^{*}\left([d \phi]_{\bmod 2 \pi}-d \phi\right),
$$

where $\phi=\operatorname{Arg} \Omega_{33}$. Here we used notations of differential forms on the lattice. For a definition of those notations see, for example, [22]. Then, worldlines of quantum $Z_{2}$ monopoles appear as the boundary of the string worldsheet:

$$
j=\delta \Sigma
$$

We expect, that the monopole currents should percolate above the transition temperature $T_{c}$ in the finite temperature model. We expect that these $Z_{2}$ monopoles resemble both $Z_{3}$ monopoles of realistic $\mathrm{PUT}_{1}$ and $Z_{2}$ monopoles of $\mathrm{PUT}_{2}$.

\section{Conclusions}

To conclude, we considered the Standard Model embedded into a unified model, the symmetry of which breaks down to the gauge group of the SM at a few Tev. During the breakdown monopoles may appear, which have masses of the order of 40 Tev. Those objects could become the lightest topologically stable magnetic monopoles. In principle, they could be detected in future during high energy collisions.

We expect that in the early Universe at the temperatures close to the temperature $T_{c}$ of the correspondent transition monopoles considered in this paper could appear in the elementary processes with a high probability. Those monopoles may even be condensed at $T>T_{c}$ as Nambu monopoles at the temperatures above the Electroweak transition temperature [15]. We present the lattice toy model for the qualitative consideration of this phenomenon.

It is worth mentioning that the presence of monopoles considered here may be excluded in the wide class of modern cosmological scenarios. In particular, inflation theory implies reheating after inflation [23]. Inflation itself washes out relic magnetic monopoles created at the temperatures of the order of GUT temperature $10^{15} \mathrm{Gev}$. In certain models the reheating temperature is thought of to be between $10^{6}$ and $10^{10} \mathrm{Gev}$ (see, for example, [24] and references therein). After reheating standard scenario of hot Universe works. The concentration of relic magnetic monopoles in the Hot Big Bang scenario was estimated in [25]. According to this estimate the concentration of magnetic monopoles created at $T \sim 1 \mathrm{Tev}$ appears to be essentially larger than the experimentally allowed one.

I kindly acknowledge private communication with M.Yu. Khlopov. This work was partly supported by RFBR grants 06-02-16309, 05-02-16306, and 07-02-00237.

\section{References}

[1] J.A. Casas, J.R. Espinosa, I. Hidalgo, hep-ph/0607279

F. del Aguila, R. Pittau, Acta Phys.Polon. B35 (2004) 2767-2780

[2] B.L.G. Bakker, A.I. Veselov, and M.A. Zubkov, arXiv:0707.1017 
[3] Martin Schmaltz, David Tucker-Smith, Ann.Rev.Nucl.Part.Sci. 55 (2005) 229-270

[4] Mark Trodden, Tanmay Vachaspati, Phys.Rev. D70 (2004) 065008

[5] Christopher T. Hill, Elizabeth H. Simmons, Phys.Rept. 381 (2003) 235-402; Erratum-ibid. 390 (2004) $553-554$

Kenneth Lane, hep-ph/0202255

R. Sekhar Chivukula, hep-ph/0011264

[6] S.Weinberg, Phys.Rev.D 13, 974, 1976

L.Susskind, Phys.Rev.D 20, 2619, 1979

[7] E.Farhi, L.Susskind, Phys.Rev.D 20, 3404, 1979

[8] Thomas Appelquist, Neil Christensen, Maurizio Piai, Robert Shrock, Phys.Rev. D70 (2004) 093010 Adam Martin, Kenneth Lane, Phys.Rev. D71 (2005) 015011

Thomas Appelquist, Maurizio Piai, Robert Shrock, Phys.Rev. D69 (2004) 015002

Robert Shrock, hep-ph/0703050

Adam Martin, Kenneth Lane, Phys.Rev. D71 (2005) 015011

[9] Andrzej J. Buras, P.Q. Hung, Phys.Rev. D68 (2003) 035015

[10] Andrzej J. Buras, P.Q. Hung, Ngoc-Khanh Tran, Anton Poschenrieder, Elmar Wyszomirski, Nucl.Phys. B699 (2004) 253

Mehrdad Adibzadeh and P.Q. Hung, hep-ph/0705.1154.

[11] C.Gardner, J.Harvey, Phys. Rev. Lett. 52 (1984) 879

Tanmay Vachaspati, Phys.Rev.Lett. 76 (1996) 188-191

Hong Liu, Tanmay Vachaspati, Phys.Rev. D56 (1997) 1300-1312

[12] B.L.G. Bakker, A.I. Veselov, and M.A. Zubkov, Phys. Lett. B 583, 379 (2004);

[13] B.L.G. Bakker, A.I. Veselov, and M.A. Zubkov, Yad. Fiz. 68, 1045 (2005).

[14] B.L.G. Bakker, A.I. Veselov, and M.A. Zubkov, Phys. Lett. B 620 (2005) 156-163.

[15] B.L.G. Bakker, A.I. Veselov, and M.A. Zubkov, Phys.Lett. B642 (2006) 147-152

[16] K.S. Babu, I. Gogoladze, and K. Wang, Phys. Lett. B 570, 32 (2003);

K.S. Babu, I. Gogoladze, and K. Wang, Nucl. Phys. B 660, 322 (2003);

[17] M.A. Zubkov, arXiv:0707.0731

[18] M.A. Zubkov, Phys. Lett. B 649, 91 (2007).

[19] Gerard 't Hooft, Nucl.Phys.B79:276-284,1974

Alexander M. Polyakov, JETP Lett.20:194-195,1974, Pisma Zh.Eksp.Teor.Fiz.20:430-433,1974

[20] Erick J. Weinberg, Nucl.Phys.B167:500,1980

[21] J.C.Pati, S.Radjpoot, A.Salam, Phys. Rev. D 17, 131 (1978)

[22] M.I. Polikarpov, U.J. Wiese, and M.A. Zubkov, Phys. Lett. B 309, 133 (1993).

[23] Lev Kofman, Andrei D. Linde, Alexei A. Starobinsky, Phys.Rev.Lett.73:3195-3198,1994.

[24] Rachel Jeannerot, Marieke Postma, JCAP 0512 (2005) 006

Masaaki Fujii, K. Hamaguchi, T. Yanagida, Phys.Rev. D63 (2001) 123513

M.Yu.Khlopov, A.D.Linde, Phys. Lett. (1984), V. 138B, PP. 265-268.

[25] M.Yu.Khlopov, A.D.Linde,Phys.Lett. (1978), V. 79B, PP. 239-242 